\title{
QUANTUM NOISE CHARACTERISTICS OF NONLINEAR OPTICAL AMPLIFIERS
}

\author{
R.W. Boyd ${ }^{a}$, G.S. AgarWal ${ }^{b}$, W.V. Davis ${ }^{a}$, A.L. Gaeta ${ }^{c}$, \\ E.M. NAGASAKo ${ }^{a}$ AND M. KAURANEN ${ }^{d}$ \\ a The Institute of Optics, University of Rochester, Rochester, NY 14627, USA \\ ' School of Physics, University of Hyderabad, Hyderabad 500 134, India \\ c School of Applied Physics, Cornell University, Ithaca, NY 14853, USA \\ ${ }^{d}$ University of Leuven, Celestijnenlann $200 \mathrm{D}, 3001$ Heverlee, Belgium
}

\begin{abstract}
Quantum mechanical arguments show that any optical amplifier must add noise to the amplified beam of light. For the case of a high gain amplifier with a coherent (Poissonian) input, the signal-to-noise ratio of the amplified beam must be at least two times smaller than that of the incident beam. We review the theoretical basis of this prediction. We also describe the results of our experimental investigations of the noise properties of optical amplifiers that utilize the nonlinear optical response of strongly driven atomic transitions.
\end{abstract}

PACS numbers: 42.50.Lc, 42.65.-k

\section{Introduction}

The subject of this paper is the signal-to-noise characteristics of optical amplifiers, and in particular the issue of how quantum noise processes influence the sensitivity of these devices [1,2]. We will be particularly interested in the special case of light amplifiers that operate by means of nonlinear optical processes in atomic vapors. However, much of the discussion presented here is more general, and can apply to a wide variety of devices, such as amplifiers that make use of different types of gain processes and of devices other than optical amplifiers such as phase conjugate mirrors [3].

It is well established that any optical amplifier must add noise to the amplified beam of light. There are several different methods for quantifying how much noise is added to the amplified beam. One standard method is to measure the signal-to-noise ratio of the incident beam and compare it to that of the amplified beam. Theoretical analysis then shows that the square of the signal-to-noise ratio of the output beam must be at least two times smaller than that of the input beam. Here we define the signal-to-noise ratio to be the ratio of the mean number of photons per mode to the rms spread in the number of photons per mode. Equivalently, one often says that the noise figure of an optical amplifier must be 
at least $3 \mathrm{~dB}$. This well known result was derived as early as 1962 by Haus and Mullen [1], and has been discussed in a more general context by Caves [2].

Let us examine the nature of the physical processes that add noise to the amplified light beam. In fact, the origin of the added noise depends upon the nature of the optical amplifier. We consider first the example of a laser amplifier. Such an amplifier contains a collection of inverted atoms. An excited atom can return to the ground state either by stimulated emission (which amplifies the incident light field) or by spontaneous emission (which adds noise to the transmitted light field). Let us contrast this situation with that of an amplifier that operates by means of a nonlinear optical interaction. For definiteness, we consider amplification by means of the parametric amplification process, in which a signal beam of frequency $\omega_{\mathbf{s}}$ interacts with a pump beam of frequency $\omega_{\mathrm{p}}$ in a nonlinear mixing crystal. In this case noise can be added to the transmitted light field by the process of parametric fluorescence, in which a single pump photon spontaneously splits into a signal and idler photon.

Since the origin of the noise and its theoretical description is different in each of these two examples of optical amplifiers, it is useful to ask if there are certain noise properties that are generally true of all optical amplifiers. In fact there are certain constraints that must be satisfied by any optical amplifier; let us see now how these constraints arise.

\section{Quantum mechanical description of an ideal optical amplifier}

Let us consider an ideal optical amplifier $[1,2]$, that is, an amplifier that adds the smallest amount of noise to the amplified beam consistent with the laws of quantum mechanics. Of course, classically, there is no need for noise to be added to the beam, and we can describe the amplifier as a device in which the incident and transmitted field amplitudes $E_{\text {in }}$ and $E_{\text {out }}$ are related by

$$
E_{\text {out }}=g E_{\text {in }}
$$

where $g$ is the amplitude gain of the amplifier.

To describe such a device quantum mechanically, we must describe the input and output fields by photon operators that we designate $\hat{a}$ and $\hat{b}$. One might think on the basis of the correspondence principle that an amplifier is a device for which $\hat{b}$ is related to $\hat{a}$ by

$$
\hat{b}=g \hat{a} \text {. }
$$

However, $\hat{a}$ and $\hat{b}$ must each obey the boson commutation relations

$$
\left[\hat{a}, \hat{a}^{\dagger}\right]=\left[\hat{b}, \hat{b}^{\dagger}\right]=1 \text {. }
$$

But we immediately see our initial assumption leads to an inconsistency, because when we calculate $\left[b, b^{\dagger}\right]$ we obtain $|g|^{2}\left[\hat{a}, \hat{a}^{\dagger}\right]=|g|^{2}$ and not 1 .

Since the assumption that $\hat{b}=g \hat{a}$ leads to an inconsistency, we instead assume that

$$
\hat{b}=g \hat{a}+\hat{L}^{\dagger}
$$

where $\hat{L}$ represents a Langevin noise operator that describes some noise source internal to the optical amplifier. Since $\hat{L}$ describes a degree of freedom different 
from that of the input field mode, we assume that $\left[\hat{a}, \hat{L}^{\dagger}\right]=[\hat{a}, \hat{L}]=0$. If we now calculate the commutator we obtain $\left[\hat{b}, \hat{b}^{\dagger}\right]=|g|^{2}\left[\hat{a}, \hat{a}^{\dagger}\right]+\left[\hat{L}^{\dagger}, \hat{L}\right]$, which is equal to unity if $\left[\hat{L}, \hat{L}^{\dagger}\right]=|g|^{2}-1$. This condition can be satisfied if we set

$$
\hat{L}=\left(|g|^{2}-1\right)^{1 / 2} \hat{c}
$$

where $\hat{c}$ is itself a boson field operator satisfying $\left[\hat{c}, \hat{c}^{\dagger}\right]=1$. Thus our quantum mechanical model of an ideal optical amplifier actually has the form shown in Fig. 1, in which the output has the form of an amplified input plus a term that

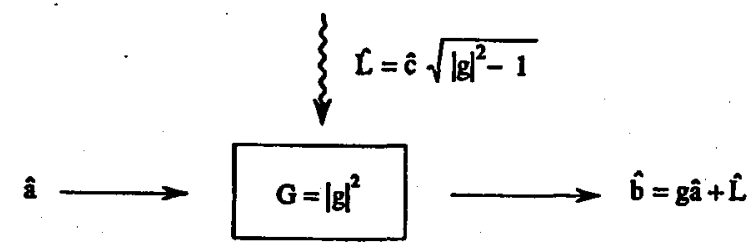

Fig. 1. Quantum mechanical model of an ideal optical amplifier.

represents some internal noise source. We make the physical assumption that the noise component fluctuates about a mean value of zero and that the noise source is in its ground state in the sense that the mean occupation number of this mode is zero. These conditions lead to the relations

$$
\langle\hat{L}\rangle=0, \quad\left\langle\hat{L}^{\dagger} \hat{L}\right\rangle=0 .
$$

We also assume that these fluctuations are uncorrelated with those of the input field so that expectation values such as $\left\langle\hat{L}^{\dagger} a\right\rangle$ also vanish.

Let us now examine some of the consequences of this model. We first introduce photon number operators for modes $\hat{a}$ and $\hat{b}$ as follows:

$$
\begin{aligned}
& \hat{n}_{a}=\hat{a}^{\dagger} \hat{a}, \\
& \hat{n}_{b}=\hat{b}^{\dagger} \hat{b}=|g|^{2} \hat{a}^{\dagger} \hat{a}+g^{*} \hat{a}^{\dagger} \hat{L}^{\dagger}+\hat{L} g \hat{a}+\hat{L} \hat{L}^{\dagger} .
\end{aligned}
$$

If we take the expectation values of these quantities and make use of the fact that $\hat{L} \hat{L}^{\dagger}=|g|^{2}-1+\hat{L} \dagger \hat{L}$, we find that

$$
\left\langle\hat{n}_{b}\right\rangle=G\left\langle\hat{n}_{a}\right\rangle+G-1,
$$

which shows that the input intensity is amplified by the factor $G=|g|^{2}$ and that $G-1$ "noise photons" are added to the output beam. We can similarly calculate the fluctuations in the output beam. We find that

$$
\left\langle\Delta \hat{n}_{b}^{2}\right\rangle \equiv\left\langle\hat{n}_{b}^{2}\right\rangle-\left\langle\hat{n}_{b}\right\rangle^{2}=G^{2}\left\langle\Delta \hat{n}_{a}^{2}\right\rangle+G(G-1)\left(\left\langle\hat{n}_{a}\right\rangle+1\right),
$$

where the first term represents the amplification of noise present in the input beam and the second term represents noise that is added to the beam by the optical amplifiers.

One standard method of characterizing the noisiness of the transmitted beam is to calculate its Fano number, which is defined by

$$
F=\frac{\left\langle\Delta \hat{n}^{2}\right\rangle}{\langle\hat{n}\rangle} \text {. }
$$


Note that $F=1$ for a beam with Poissonian photon fluctuations, and that $F$ exceeds unity for noisier (i.e., super-Poissonian) beams. If we calculate the Fano number of the output beam we find that

$$
F_{b}=\frac{G^{2}\left\langle\Delta \hat{n}_{a}^{2}\right\rangle+G(G-1)\left(\left\langle\hat{n}_{a}\right\rangle+1\right)}{G\left(\hat{n}_{a}\right\rangle+G-1} .
$$

In the limit of a high gain amplifier $(G \gg 1)$ with a strong input beam $\left(\left\langle\hat{n}_{a}\right\rangle \gg 1\right)$, we find that

$$
F_{b}=G\left(F_{a}+1\right)
$$

which by assumption is much greater than unity, even in the limiting case in which the input field is in a number state and $F_{a}$ vanishes. We thus find that the output of a high gain optical amplifier is extremely noisy in the sense that $F_{b} \gg 1$, that is, that the photon number fluctuations are much larger than $\sqrt{\left\langle\hat{n}_{b}\right\rangle}$.

It is also useful to describe the properties of optical amplifiers in terms of signal-to-noise ratios. We define the signal-to-noise ratios of the input and output beams by

$$
\begin{aligned}
& (S / N)_{a}=\left\langle\hat{n}_{a}\right\rangle /\left[\left\langle\Delta \hat{n}_{a}^{2}\right\rangle\right]^{1 / 2}, \\
& (S / N)_{b}=G\left\langle\hat{n}_{a}\right\rangle /\left[\left\langle\Delta \hat{n}_{b}^{2}\right\rangle\right]^{1 / 2} .
\end{aligned}
$$

Note that we take the "signal" part of the output to be $G\left\langle\hat{n}_{a}\right\rangle$, that is, we do not include the contribution of $G-1$ to $\left\langle\hat{n}_{b}\right\rangle$ which represents the "background". We next define the noise figure $\mathcal{F}$ of the amplifier as the factor by which the square of the signal-to-noise ratio is reduced by the amplification process, that is

$$
\mathcal{F} \equiv \frac{(S / N)_{a}^{2}}{(S / N)_{b}^{2}}=\frac{\left\langle\Delta \hat{n}_{b}^{2}\right\rangle}{G^{2}\left\langle\Delta \hat{n}_{a}^{2}\right\rangle}
$$

By introducing expression (10) for $\left\langle\Delta \hat{n}_{b}^{2}\right\rangle$, we find that

$$
\mathcal{F}=1+\left(1-\frac{1}{G}\right) \frac{\left\langle\hat{n}_{a}\right\rangle+1}{\left\langle\Delta \hat{n}_{a}^{2}\right\rangle} .
$$

For a high gain $(G \gg 1)$ amplifier with a strong $\left(\left\langle n_{a}\right\rangle \gg 1\right)$ input, we obtain

$$
\mathcal{F}=1+\frac{\left\langle\hat{n}_{a}\right\rangle}{\left\langle\Delta \hat{n}_{a}^{2}\right\rangle}
$$

if in addition the input fluctuations are Poissonian, we obtain the well known result quoted above in the introduction,

$$
\mathcal{F}=2 .
$$

We have defined the noise figure as we did in Eq. (16) as the square of the ratio (rather than the ratio directly) of the input and output signal-to-noise ratios because in the present paper we define the signal-to-noise ratio in terms of photon number fluctuations and not, as is commonly done, in terms of field fluctuations. 


\section{Optical attenuator}

The properties of an ideal attenuator can be deduced by a calculation analogous to the one just described. We find that the output ficld is related to the input by

$$
\hat{b}=t \hat{a}+\hat{L}
$$

where

$$
\hat{L}=\sqrt{1-|t|^{2}} \hat{c},
$$

where $\hat{c}$ again describes a boson field (i.e., $\left[\hat{c}, \hat{c}^{\dagger}\right]=1$ ) and where $t$ denotes the amplitude transmission of the attenuator. We find through direct computation that the Fano number of the output ficld is given by

$$
F_{b}=T F_{a}+(1-T)
$$

where $T=|\ell|^{2}$. Note that $F_{b} \rightarrow 1$ for $T \ll 1$. Thus the output of a hight loss attenuator is Poissonian for any type of input fluctuations. We can also calculate the noise figure, defined as above in Eq. (16). We find that

$$
\mathcal{F}=1+\frac{\left\langle\hat{n}_{a}\right\rangle}{\left\langle\Delta \hat{n}_{a}^{2}\right\rangle}\left(\frac{1}{T}-1\right)
$$

and that for a Poissonian input beam

$$
\mathcal{F}=\frac{1}{T} \text {. }
$$

This equation shows how rapidly the signal-to-noise ratio is degraded by the random removal of photons from the light beam.

\section{Non-ideal amplifiers}

Our discussion up to now lias dealt with the noise properties of ideal amplifiers. In fact, many amplificrs arc non-idcal in that they produce more noise than that required by the laws of quantum mechanics.

As a simple example of a non-ideal amplifier, we consider the situation illustrated in Fig. 2 in which a $10 \times$ ideal attenuator placed in front of a $100 \times$ ideal amplifier. If a strong coherent input beam falls onto such a combination, we find

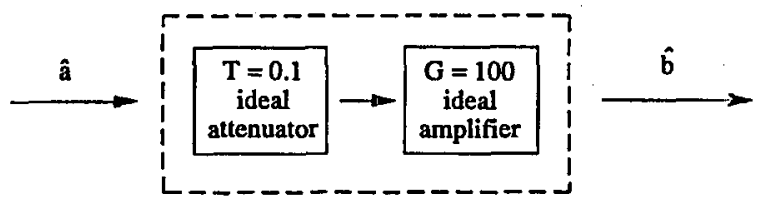

Fig. 2. Construction of a non-idcal amplificr from two ideal components.

by direct calculation using the formulas described above that the combination has a noise figure of 19.9. Conversely, a 10x ideal amplifier has a noise figure of 1.9. This simple example shows how easy it is to construct a non-ideal amplifier even 
using ideal components. It also provides quantitative confirmation of the intuitive thought that it is foolish to attenuate a beam before amplifying it.

Laser amplifiers are oftcn non-ideal. For example, three-level laser amplifiers are always non-ideal, because the population inversion is necessarily less than $100 \%$. Because of this, emission and absorption processes occur simultaneously, and both of them contribute to the noise, whereas only emission contributes to amplification. Although three-level laser amplifiers cannot be ideal, noise figurcs as low as $4 \mathrm{~dB}$ have been measured for erbium doped fiber amplifiers [4]. On the other hand, four-level laser amplifiers can be nearly ideal because the population inversion is essentially $100 \%$.

Another commonly employed method of assessing the noise properties of an amplifier is by means of the noise temperature. The noise temperature is defined in the following manner. The mean number of photons per mode leaving an optical amplifier can be represented as

$$
\left\langle\hat{n}_{b}\right\rangle=G\left\langle\hat{n}_{a}\right\rangle+N_{f}(G-1) .
$$

IIere the first term represents the amplified input signal and the second term the number of noise photons added to the output beam. Ilere $N_{f}$ represents an excess noise factor that tells how much noisier the amplifier is than an ideal amplifier for which $N_{f}=1$. Note that for $G \gg 1$ this much noise would be produced by an effective background input of $N_{f}$ photons per mode, and an input of this level would be provided by a thermal source of temperature $T_{N}$ given by

$$
T_{N}=\frac{h \nu}{k \ln \left(1+\frac{1}{N_{s}}\right)} .
$$

For an ideal amplifier $\left(N_{f}=1\right)$ this result takes on the wcll known form

$$
T_{N}=h \nu / k \ln 2 .
$$

For visible light $T_{N}$ is of the order of $10000 \mathrm{~K}$.

\section{Amplification by the response of a strongly driven atom}

We now consider optical amplifiers that operate by means of the nonlinear response of a strongly driven atomic system. We consider an atomic vapor that is simultaneously irradiated by a strong pump wave at frequency $\omega$ and a weak signal wave detuned from the pump wave by the amount $\delta$, as shown in Fig. 3.

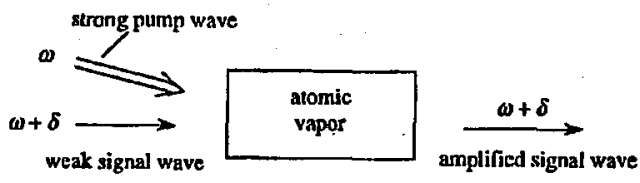

Fig. 3. Light amplifier based on a strongly driven atom.

This type of amplifier operates by means of the modification of the atomic encrgy structure in the presence of the strong pump wave; this modification is 

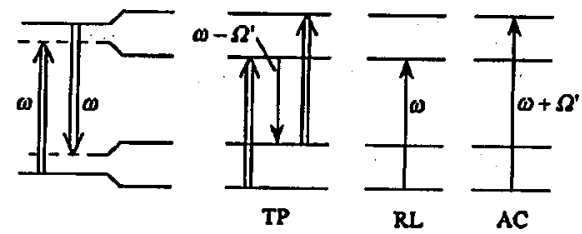

Fig. 4. Modification of the atomic energy-level structure by the dynamic Stark effect. $\mathrm{TP}=$ three-photon feature, $\mathrm{RL}=$ Rayleigh feature, $\mathrm{AC}=$ Stark-shifted feature.

often known as the dynamic Stark effect and is illustrated in Fig. 4. As a result of the coherent oscillation of populations between the upper and lower atomic states, the atom takes on the illustrated four-level structure [5]. The doublet separation is equal to the Rabi floppy frequency which is given by

$$
\Omega^{\prime}=\left(\Omega^{2}+\Delta^{2}\right)^{1 / 2}, \quad \Omega=\mu E_{0} / h, \quad \Delta=\omega-\omega_{b a} .
$$

This four-level system is secn to possess three resonances at frequencies $\omega, \omega+\Omega^{\prime}$, and $\omega-\Omega^{\prime}$.

Mollow [6] has calculated the probe absorption spectrum experienced by the signal wave in propagation through such a strongly driven atomic vapor and obtains the result shown in Fig. 5. The spectrum is seen to contain 3 features,

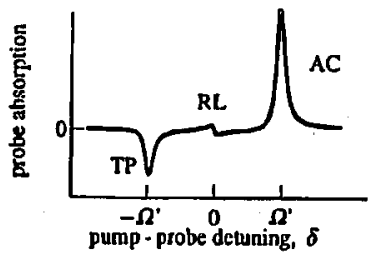

Fig. 5. Absorption spectrum of a strongly driven atomic system.

each of which corresponds to one of the resonances shown in Fig. 4. Two of the features show negative absorption, that is, gain. The positive-going feature corresponds to the shifted absorption of the atom. The central feature is a form of stimulated Rayleigh scattering. The other Rabi-sideband shows gain by means of the three-photon effect; the atom makes a transition from the lowest dressed level to the highest by the simultaneous absorption of two-pump photons and the emission of a Rabi sideband photon. Since the three-photon feature typically has the largest gain, most of our experimental work has stressed the three-photon feature.

We have recently been engaged in an experimental investigation of atomic vapor nonlinear optical amplifiers [7]. Some of the properties of these amplifiers that make them desirable for practical applications are as follows:

(1) Single-pass amplifications of greater than 100 have been achieved using a 1-cm-long cell.

(2) This type of amplifier preserves the wavefront structure of the incident signal beam, rendering it useful for applications such as a preamplifier in a coherent imaging system. 
(3) The amplificr can amplify even beams that are rapidly temporally modulated (> $1 \mathrm{GHz}$ ), allowing it to be used for example in high speed telecommunications systems.

(4) The amplifier possesses a $100 \%$ duty factor, because it can be pumped by $\mathrm{cw}$ lasers.

\section{Noise properties of amplification by strongly driven atoms}

Recently we have performed a thcoretical study of the noise properties of atomic vapor nonlinear optical amplifiers [8]. This calculation was based on a theoretical formalism developed by G.S. $\Lambda$ garwal and makes use of master equation techniques. Wc find that the number of noise photons added to the beam is larger than the number for an ideal amplifier by an excess noise factor $N_{f}$ and that noise figure $\mathcal{F}$ exceeds the idcal-amplifier value of 2 by the same factor. We find that the stimulated Rayleigh feature never acts as an ideal amplifier and is always at least 4 times noisier than ideal, that is, the excess noise factor $N_{f}$ is never smaller than 4. IIowever, the three-photon feature can approach the case of an ideal amplifier $\left(N_{f}=1\right)$ in the limit of a large pump detuning from resonance for a medium in which the broadening is radiative rather than collisional, that is, for the case in which $T_{2}=2 T_{1}$.

Some of our preliminary experimental results obtained using a potassium atomic vapor for the three-photon gain mechanism are shown in Fig. 6. We plot

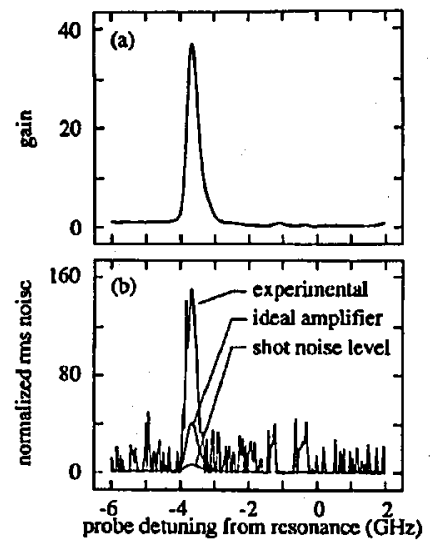

Fig. 6. Measured gain (a) and noise (b) spectra of the three-ploton gain mechanism for a potassium atomic vapor.

the measured gain and the normalized rms noise as functions of the probe detuning from the atomic resonance frequency. We sce a maximum gain of $\approx 40$ at the peak of the three-photon feature. Also shown is the measured noise and in comparison the prediction for an ideal amplifier, which itself is much larger than the shot noise level of the amplified beam. This experiment was conducted at a number density of $3 \times 10^{13} \mathrm{~cm}^{-3}$, and a pump detuning of $\approx 2 \mathrm{GIIz}$. Under these conditions, the theory 
described above predicts that the amplifier should be esscntially ideal. However, the measured noise is almost three times larger than that expected of an ideal amplifier. We suspect that the origin of this discrepancy is the effects of atomic motion, which are not included in the theoretical analysis presented above. We are presently in the process of modifying the theory to include these effects.

\section{Noise properties of nonlincar optical beam propagation}

We have presently performed an experiment in which we observed that excess noise can be added to a single beam of light as it propagates through an atomic vapor [9]. In particular, we find that if an intense, shot noise limited beam is incident on an atomic vapor, the transmitted beam can become very noisy.

(a)

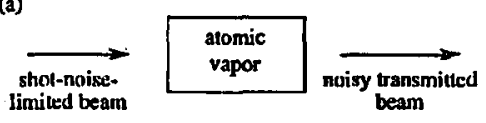

(b)

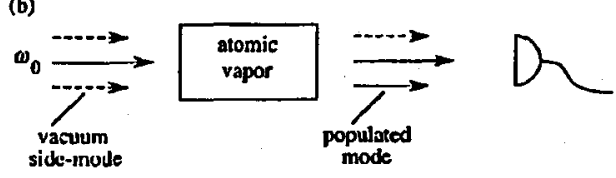

Fig. 7. (a) $\mathrm{A}$ strong shot-noise-limited beam can become very noisy in passing through an atomic vapor. (b) Model describing the origin of the added noisc. The two populated modes beat together at the photodetector.

This effect and our explanation of it are shown in Fig. 7. The incident beam actually consists of an intense populated ficld mode and its vacuum sidemodes. If the pump intensity is high enough, one of these sidemodes will be at a frequency where gain is present, and real photons will be produced at the output as a consequence of the properties of optical amplifiers that are described above. These two field modes can then beat together at the photodetector to produce noise in the photocurrent.

Some of our experimental results are shown in Fig. 8. In this experiment we have actually used separate pump and probe beams of the same frequency to avoid the problem of having an intense field fall onto the photodiode. As we tune the laser frequency through line center, we see that a dip occurs in the transmitted intensity. Nonetheless, the noise increases except at the very centcr of the line as a result of the noise mechanism described above. Shown also are the results of a theoretical calculation based on the master equation method described above.

Note that this noise mechanism is present whenever an intense laser beam propagates through an atomic vapor, and that it can degrade the amount of squeezing that can be achieved in noise reduction schemes that utilize atomic vapors. In fact, we suspect that this noise mechanism could explain why squeezing experiments have been much more successful in parametric mixing crystals and in optical 


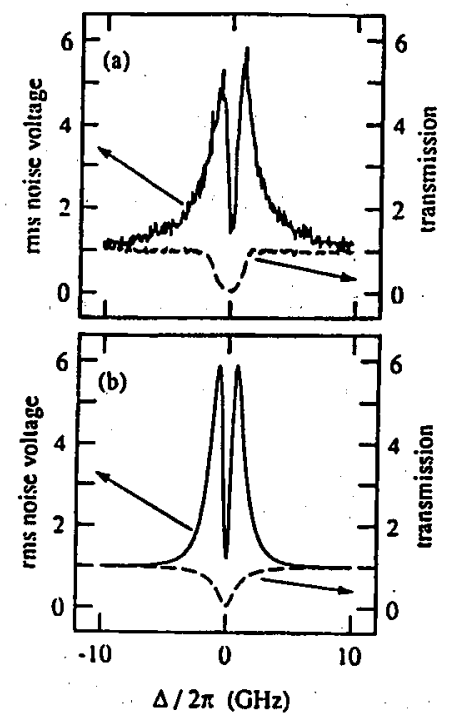

Fig. 8. Experimental measurements (a) and theoretical predictions (b) for the properties of a beam transmitted by an atomic vapor.

fibers than in atomic vapors. We are presently working on repeating this experiment in the cleaner, single-beam version.

\section{Acknowledgments}

The portion of the work performed at the University of Rochester was supported by the U.S. Army Research Office under a University Research Initiative. We are also grateful for the support through NSF travel grant INT 9017532 which allowed one of us (G.S.A.) to travel to Rochester to work on this project.

\section{References}

[1] H.A. Haus, J.A. Mullen, Phys. Rev. 128, 2407 (1962).

[2] C.M. Caves, Phys. Rev. D 26, 1817 (1982).

[3] A.L. Gaeta, R.W. Boyd, Phys. Rev. Lett. 60, 2618 (1988).

[4] Y. Yamamoto, T. Mukai, Opt. Quantum Electron. 21, S1 (1989).

[5] R.W. Boyd, Nonlinear Oplics, Academic, New York 1992.

[6] B.R. Mollow, Phys. Rev. A 5, 2217 (1972).

[7] M.T. Gruneisen, K.R. Mac Donald, R.W. Boyd, J. Opt. Soc. Am. B 5, 123 (1988).

[8] A.L. Gaeta, R.W. Boyd, G.S. Agarwal, Phys. Rev. A 46, 4271 (1992).

[9] M. Kauranen et al., to be published. 